\title{
Reply to: Accuracy and reproducibility of the ETDRS visual acuity chart: methodological issues
}

\author{
Reuben R. Shamir ${ }^{1}$ - Yael G. Friedman ${ }^{2} \cdot$ Leo Joskowicz $^{1} \cdot$ Michael Mimouni $^{2}$ • \\ Eytan Z. Blumenthal ${ }^{2}$
}

Received: 20 June 2016 / Accepted: 21 June 2016 /Published online: 30 June 2016

(C) Springer-Verlag Berlin Heidelberg 2016

We thank Drs. Sabour and Ghassemi for their thoughtful comment. Indeed, in most (if not all) studies on visual acuity (VA), since a gold standard is lacking, data can be summarized only in terms of reproducibility (and/or variability), and never as accuracy. However, since this study is a computer simulation, we were able to initially define a true VA, and thereafter run the simulation multiple times and obtain measured VAs [1,2].

In this study, accuracy was defined as the difference between the mean of the measurements and the reference (true) value for each chart. Reproducibility was defined as test-retest variability between consequent runs. Figure 3 in the article depicts improved accuracy up to seven characters, flattening thereafter, and shows that separate curves exist for odd vs. even numbers of characters per row [2].
We fully agree that accuracy and reproducibility are separate issues, and feel that this is one of the few studies on VAalthough not without limitations - permitting a glance at accuracy, rather than being limited to reproducibility.

\section{References}

1. Shamir RR, Friedman Y et al (2016) Comparison of snellen and early treatment diabetic retinopathy study charts using a computer simulation. Int J Ophthalmol 9(1):119-123

2. Shamir RR, Friedman YG et al (2016) The influence of varying the number of characters per row on the accuracy and reproducibility of the ETDRS visual acuity chart. Graefes Arch Clin Exp Ophthalmol 254(5):971-976
Eytan Z. Blumenthal

e_blumenthal@ rambam.health.gov.il

1 School of Engineering and Computer Science, Hebrew University, Jerusalem, Israel

2 Department of Ophthalmology, Rambam Medical Center, P.O.B 9602, Haifa 31096, Israel 